\title{
A Unique Property of a Plasma Proteoglycan, the C1q Inhibitor An Anticoagulant State Resulting from Its Binding to Fibrinogen
}

\author{
Dennis K. Galanakis and Berhane Ghebrehiwet \\ Blood Bank, University Hospital, State University of New York, Stony Brook, New York 11794
}

\begin{abstract}
The C1q inhibitor, C1qI, an $\sim 30-k D$ circulating chondroitin-4 sulfate proteoglycan, displayed concentration-dependent prolongation of plasma and fibrinogen solution clotting times. Under factor XIIIa catalyzed cross-linking conditions and maximum C1qI concentrations, minor amounts of clot formed displaying complete $\boldsymbol{\gamma}-\boldsymbol{\gamma}$ dimer formation but virtually no $\alpha$-polymer formation. The anticoagulant effect was undiminished by its binding to $\mathrm{Clq}$, by increased ionic strength, and by $\mathrm{CaCl}_{2}$, but was abolished by incubation of $\mathrm{ClqI}$ with chondroitinase ABC. ${ }^{125}$ I-labeled C1qI bound to immobilized fibrinogen, fibrin monomer, fibrinogen plasmic fragments $D_{1}$ and $E$, and fibrin polymers. Occupancy on the $\mathrm{E}$ domain required uncleaved fibrinopeptides together with another structure(s), and it did not decrease binding of thrombin to fibrinogen. Occupancy on the D domain did not decrease the fibrinogen binding to fibrin monomer. We conclude that the $\mathbf{E}$ domain occupancy impaired fibrinopeptide cleavage, and occupancy on the $D$ domain impaired polymerization, both steric hindrance effects. C1qI binding to fibrinogen explains at least in part the well-known fibrin(ogen) presence in immune complex-related lesions, and the fibrinogen presence in vascular basement membranes and atheromata. We postulate that fibrin binding by resident basement membrane proteoglycans provides dense anchoring of thrombus, substantially enhancing its hemostatic function. (J. Clin. Invest. 1994.93:303-310.) Key words: anticoagulant $\cdot \mathrm{Clq}$ inhibitor • fibrin • fibrinogen • proteoglycan
\end{abstract}

\section{Introduction}

Fibrinogen is a plasma glycoprotein containing three pairs of polypeptide chains $\mathrm{A} \alpha, \mathrm{B} \beta$, and $\gamma$ interlinked by disulfide bridges which are clustered in three regions, the central or $\mathrm{E}$ and two outer or D domains ( 1 ). It is converted to fibrin monomer by cleavage of two small amino-terminal peptides, $A$ and $\mathrm{B}$, by thrombin-A from its $\mathrm{A} \alpha$ and $\mathrm{B}$ from its $\mathrm{B} \beta$ chain, respectively. Fibrin monomers spontaneously polymerize to form the gel matrix, which is further stabilized by the formation of covalent links catalyzed by factor XIIIa. Polymerization is initiated by the binding of one fibrin $\mathrm{E}$ domain to the $\mathrm{D}$ domains of two other fibrin molecules thus forming a two-molecule-thick strand, the protofibril.

\footnotetext{
Address correspondence to Dr. Dennis K. Galanakis, Blood Bank, University Hospital, SUNY, Stony Brook, NY 11794.

Received for publication 12 March 1993 and in revised form 22 July 1993.
}

J. Clin. Invest.

(c) The American Society for Clinical Investigation, Inc.

$0021-9738 / 94 / 01 / 0303 / 08 \quad \$ 2.00$

Volume 93, January 1994, 303-310
Glycosaminoglycans (GAG) ${ }^{1}$ are present in blood in concentrations of $\sim 5 \mu \mathrm{g} / \mathrm{ml}$ (2). One fourth or fewer of these circulate in free form, and the remainder are bound to unidentified proteins. GAG heterogeneity (3), their presence in numerous sites (4) such as cell membranes and basement membranes, and their release from cells in culture $(3,5)$ suggest multiple sites of origin. The major form in human plasma (2, 4 ) is the low sulfated chondroitin-4 sulfate proteoglycan (C4SP), which consists of repeating disaccharides of glucuronic acid- $N$-acetyl-galactosamine, generally bound to the hydroxyl group of a serine residue of a core peptide to give the sequence (disaccharide)nGlcUA-Gal-Gal-Xyl-O-Ser. These repeating disaccharides are sulfated to a variable degree, a variation that modifies interactions (5) with other molecules. The discovery that a polyanionic molecule(s) was associated with isolated $\mathrm{Clq}(6)$, the first component of complement, led to its identification (4) as a heterogeneous population of C-4SP that displayed potent inhibition of the hemolytic activity of Clq (4), whether it was isolated from serum $(4,6)$ or from lymphocyte membranes (7). The known interaction of fibrin with tissue glycosaminoglycans $(8,9)$, and the increased circulating concentrations of proteoglycans in certain pathologic states $(2)$ led us to examine the possible interactions between $\mathrm{ClqI}$ and fibrinogen/fibrin [ fibrin(ogen)]. Initial results disclosed binding to and an anticoagulant property against fibrin(ogen) by $\mathrm{ClqI}$. This formed the subject of the present investigations.

\section{Methods}

Fibrinogen, fibrin, and plasmic fragments. Fibrinogen was isolated and its concentration was determined as described (10). Fibrin monomer preparation and polymerization (11) and SDS-PAGE (12) were performed as described. Polymerization rates were computed from the time course of clot absorbance at $350 \mathrm{~nm}$, and clot turbidity maxima were obtained as described (11). Batroxobin and human thrombin were kind gifts from Dr. K. Stocker (Pentapharm Ltd., Basel, Switzerland) and Dr. J. Jesty (Health Sciences Center, SUNY, Stony Brook, NY), respectively. Hirudin (Sigma Chemical Co., St. Louis, MO) and D-phenylalanyl-prolyl-arginyl chloromethyl ketone (P-PACK) (Calbiochem Corp., La Jolla, CA) were used as provided. Plasmic fragments $D_{1}$ and $E$ were isolated and their concentrations were measured by procedures previously applied (11). Plasma coagulability was measured by adding $\sim 50-200 \mu \mathrm{g}$ of ${ }^{125} \mathrm{I}$-labeled fibrinogen (11) per milliliter of plasma, and by obtaining thrombin $(1 \mathrm{U} / \mathrm{ml})$-induced clots, at ambient and then at $4^{\circ} \mathrm{C}$ temperatures for $1 \mathrm{~h}$ and several hours, respectively. The counts per minute $(\mathrm{cpm})$ of synerized clots and their supernatants ( $>90 \%$ of original volume) were obtained in a $\gamma$ counter. ${ }^{125} \mathrm{I}$ labeled ClqI ( vide infra) clot binding was obtained either by incorporation before clotting or by immersing the clot in the ClqI solution overnight. To obtain cross-linked clots the buffer, Tris- $\mathrm{HCl}, \mathrm{pH} 7.4$,

1. Abbreviations used in this paper: C-4SP, chrondroitin-4 sulfate proteoglycans; GAG, glucosaminoglycans; KPTI, Kunitz pancreatic trypsin inhibitor; P-PACK, D-phenylalanyl-prolyl-arginyl chloromethyl ketone. 
$\mu=0.15$ contained factor XIII $5-40 \mathrm{nM}, 2-30 \mathrm{mM} \mathrm{CaCl}_{2}, 0.1 \mathrm{U} / \mathrm{ml}$ human thrombin, and Kunitz pancreatic trypsin inhibitor KPTI (FBA Pharmaceuticals, West Haven, CT) $100 \mathrm{U} / \mathrm{ml}$. In some experiments, clots were obtained by dilution of preformed fibrin monomer solutions with this buffer (11) in the presence or absence of ClqI. Under these conditions factor XIIIa activity measured by the monodansyl cadaverine assay ( 13$)$ was unaffected by $\mathrm{ClqI}(n=2)$. Chondroitinase $\mathrm{ABC}$, which cleaves galactosaminoglycan side chains from C-4SP, and chondroitin sulfate A (a core-free C-4SP carbohydrate side chain component) were used as supplied (Sagakaku America Inc., Rockville, MD). Fibrinopeptide measurements were performed by HPLC (14). For binding measurements, ClqI was added to solutions containing 3-5 $\mu \mathrm{g} / \mathrm{ml}$ of $\mathrm{A}+\mathrm{B}$ peptides (each in roughly equal concentrations), vortex-mixed, and filtered after 5-10 min via a $10,000 \mathrm{~mol}$ wt cutoff (Millipore Corp., Bedford, MA) filter. This removed C1qI which otherwise appeared as a single protein peak near the start of the HPLC acetonitrile elution.

ClqI isolation and related analyses. C1qI was isolated essentially as described (6), and its electrophoretic (SDS-PAGE) bands could not be stained with Coomassie Brilliant Blue dye. It was subfractionated by size exclusion chromatography to obtain the active moiety (15). The ClqI concentration was determined by using a Bosch and Loemb (Leica Inc., Buffalo, NY) refractometer; thereafter, the calculated extinction coefficient $\mathrm{A}(1 \%, 1 \mathrm{~cm}$ at $280 \mathrm{~nm})$ of the active chromatographic isolate, 2.8 (range 2.6-3.0, n= 3) was used. The isolation procedure can be summarized as follows. Serum containing $1 \mathrm{mM}$ EDTA, pH 7.5, $\mu=0.16$, was incubated at $37^{\circ} \mathrm{C}$ for $\sim 10 \mathrm{~min}$ to dissociate $\mathrm{Clq}$ from $\mathrm{Cl} \mathrm{r}$ and $\mathrm{Cls}$. This was diluted to a final concentration of $\mu=0.04$, followed by stirring at $4^{\circ} \mathrm{C}$ for at least $20 \mathrm{~min}$ to precipitate $\mathrm{Clq}$. The centrifuged $\left(4^{\circ} \mathrm{C}\right)$ precipitate was washed thrice in buffer containing $5 \mathrm{mM}$ EDTA to remove contaminants, and allowed to dissolve in $0.7 \mathrm{M} \mathrm{NaCl}$ overnight. Material remaining insoluble was discarded, and the remaining solution was dialyzed against 100 mM EDTA, pH $5, \mu=0.078$. The precipitate was removed by centrifugation $\left(16,000 \mathrm{~g}, 30 \mathrm{~min}, 4^{\circ} \mathrm{C}\right)$, washed twice in cold buffer excess, and dissolved in $300 \mathrm{mM} \mathrm{NaCl}, 5 \mathrm{mM}$ EDTA, pH 7.5. This solution, further centrifuged to remove insoluble material, was dialyzed in barbital buffer, $\mu=0.5$, pH 8 , and $1 \mathrm{mM}$ each of $\mathrm{CaCl}_{2}$, and $\mathrm{MgCl}_{2}$. The solution was subjected to Con A-Sepharose (Pharmacia-LKB, Piscataway, $\mathrm{NJ}$ ) chromatography, harvesting the inhibitor in the unbound eluate (and $\mathrm{Clq}$ by subsequent elution with $10 \%$ methyl- $\alpha$-D-mannopyranoside, Sigma Chemical Co.). The unbound fraction was subjected to HPLC (14) exclusion chromatography (TSK columns, Beckman Instruments, Inc., Palo Alto, CA ), $\mathrm{pH} \mathrm{7.4, \mu}=0.15$. The protein peak displaying inhibition against the $\mathrm{Clq}$ hemolytic activity amounted to $\sim 15 \%$ of total protein and was examined for its effect on fibrinogen clotting. Possible contaminant Con A contributing to the effects on fibrin (ogen) was excluded by one ClqI preparation that was exposed overnight to solid phase (Sepharose-bound, Sigma Chemical Co.) anti-Con A IgG antibody. Its inhibition against fibrin gelation was undiminished by this exposure. For C1qI digestion, chondroitinase $\mathrm{ABC}$ was added to a solution of radiolabeled or unlabeled C1qI, 5-20 $\mu \mathrm{g} / \mathrm{ml}, \mathrm{pH} 8$, to achieve $30 \mathrm{mU} / \mathrm{ml}$ and incubated overnight, $37^{\circ} \mathrm{C}$. The mixture also contained $10 \mu \mathrm{g} / \mathrm{ml}$ pepstatin, $20 \mu \mathrm{g} / \mathrm{ml}$ leupeptin (Calbiochem Corp.), $1 \mathrm{mM}$ benzamidine, and $2 \mathrm{mM} o$-phenanthroline (Sigma Chemical Co.). The mixture was heated at $90^{\circ}$ for $1 \mathrm{~min}$ and tested for fibrinogen clotting effects either directly or after dialysis against the testing buffer. This heat exposure of untreated ClqI control did not decrease its fibrin binding or its effect against fibrin polymerization $(n=2)$. Lack of degradation of fibrin was ascertained by demonstrating no change in the polymerization rate, clot turbidity, and its SDS-PAGE bands (11).

Assay of ClqI activity. Solutions containing C1qI were tested by a hemolytic assay using C1q-deficient or C1q-depleted (C1qD, Quidel, San Diego, CA) serum as described $(16,17)$. Briefly, isolated C1q $(5-10 \mu \mathrm{g} / \mathrm{ml})$ was mixed with various concentrations of ClqI in a final volume of $300 \mu \mathrm{l}$ of veronal buffer, $\mathrm{pH} 7.5$, adjusted to $\mu=0.15$ with $\mathrm{NaCl}$, containing $\mathrm{CaCl}_{2} 1.5 \mathrm{mM}, \mathrm{MgCl}_{2} 5 \mathrm{mM}$, and $0.1 \%$ gelatin).
After $30 \mathrm{~min}$ at $37^{\circ} \mathrm{C}, 10 \mu \mathrm{l}$ of ClqD serum and $200 \mu \mathrm{l}$ of $1.5 \times 10^{-8} / \mathrm{ml}$ sheep erythrocytes sensitized with specific antibody were added and incubated for a further $60 \mathrm{~min}$ at $37^{\circ} \mathrm{C} ; 1 \mathrm{ml}$ of cold buffer was then added to stop the reaction and the hemoglobin released in the supernatant was assayed spectrophotometrically at $412 \mathrm{~nm}$. The ClqI activity was computed as percentage of the Clq control hemolytic activity.

Production and isolation of antibody. Monospecific polyclonal antibody was raised in rabbits by multiple subcutaneous and deep intramuscular injections of $\sim 100-200 \mu \mathrm{g} / \mathrm{ml} \mathrm{ClqI}$ suspended in complete Freund's adjuvant which had been diluted with incomplete adjuvant $1: 2$. The second set of injections was given 2 wk later by the same route, with ClqI suspended in complete/incomplete adjuvant ratio of 1:4 Subsequent injections were made in incomplete adjuvant at 2 wk intervals; 6 wk after the fourth injection date a final injection was given and the rabbits were bled $10 \mathrm{~d}$ later. IgG was isolated using the ImmunopPure A/G IgG purification kit (Pierce Chemical Co., Rockford, IL) according to the manufacturer's procedure, and was assayed by radial immunodiffusion. A single precipitin arc was shown against $\mathrm{ClqI}$ and there was no cross-reactivity with $\mathrm{Clq}$ and with $\mathrm{Con} \mathrm{A}$. The isolated IgG neutralized the capacity of $\mathrm{ClqI}$ to inhibit the $\mathrm{Clq}$ hemolytic activity.

Radioiodination and measurements. ${ }^{125}$ I-labeling was carried out (11) as described, and specific activities were $>60 \times 10^{3} \mathrm{cpm} / \mathrm{ng}$ protein. Autoradiograms and densitometry were obtained on dried polyacrylamide gels by using X-ray film (Eastman Kodak Co., Rochester, NY) and a (Pharmacia-LKB) laser densitometer equipped with a recorder, respectively.

Binding assays. These were carried out using buffer, pH 7.4, $\mu=0.15$, containing KPTI $100 \mathrm{U} / \mathrm{ml}$ throughout. Fibrinogen solution, $50 \mu \mathrm{g} / \mathrm{ml}$, $\mathrm{pH} 7.4$, was added to polystyrene (Immulon Removawell, Dynatech Laboratories, Alexandria, VA) miniature test tubes (i.e., removable microwells or microplates) in 60 - or 70- $\mu$ l aliquants and left covered in a moist chamber overnight at ambient temperature or for $2 \mathrm{~h}$ at $37^{\circ} \mathrm{C}$. The solution was then replaced with human serum albumin $50 \mu \mathrm{g} / \mathrm{ml}$ for at least several hours; it was then removed and the tube washed thrice with excess buffer; the desired ligand was then added and after incubation this washing procedure was repeated, but the final wash was performed five times and the buffer contained $0.05 \%$ Tween 20 . Residual wash liquor was carefully removed using a micropipette. Under these conditions $1.4 \mathrm{pM}$ of fibrinogen and $0.4 \mathrm{pM} \mathrm{Clq}$ bound to each microwell, calculated from a 1:50 mixture of $\left({ }^{125} \mathrm{I}-\mathrm{la}-\right.$ beled / unlabeled protein, $n=7$ ). To prepare fibrin monomer thrombin $0.1 \mathrm{U} / \mathrm{ml}$ or batroxobin $0.5 \mathrm{U} / \mathrm{ml}$, containing $\mathrm{KPTI}, 200 \mathrm{U} / \mathrm{ml}$, was left for several or more hours. This was then replaced for 30-60 min with enzyme neutralizing buffer $(50 \mu \mathrm{g} / \mathrm{ml}$ albumin $)$ containing PPACK $50 \mathrm{nM}$ or hirudin $5 \mathrm{U} / \mathrm{ml}$ to neutralize thrombin, or PMSF 100 $\mathrm{nM}$, to neutralize batroxobin.

\section{Results}

\section{Investigations of whole ClqI}

In pilot experiments, several $\mathrm{ClqI}$ isolates separated from $\mathrm{Clq}$ by the Con A chromatographic procedure prolonged the thrombin clotting times of plasma and of isolated fibrinogen solutions. For example, one ClqI isolate (at $38 \mu \mathrm{g} / \mathrm{ml}, \mathrm{pH} 7.4$ ) decreased the thrombin $(0.2 \mathrm{U} / \mathrm{ml})$ induced polymerization rate of fibrin $(2 \mu \mathrm{M}, \mathrm{pH} 7.4)$ from 2.6 to $1.38\left(\times 10^{-3} / \mathrm{s}\right.$ ( see Methods). A similar inhibition was demonstrated by soluble fibrin monomer induced to polymerize $(n=4)$. Addition of ClqI to plasma in further investigations resulted in invariable delay in clotting times which was concentration dependent, demonstrable in either heparin or citrate-anticoagulated plasma, in the presence (Table I) or absence of $\mathrm{CaCl}_{2}$, and whether clotting was induced by thrombin, batroxobin, or ancrod (not shown). Clots formed and were partly or fully soluble in urea $(n=4)$. SDS-PAGE analyses $(n=2)$ disclosed the 
Table I. Effect of Three Different Concentrations of a Single Preparation of Whole or Unchromatographed ClqI on Thrombin Times of Plasma

\begin{tabular}{ccccc}
\hline \multicolumn{1}{c}{ ClqI $(\mu g / m l)$} & 5 & 8 & 26 & Control \\
\hline Thrombin time $(s)$ & 16.8 & 26.6 & $>300$ & 6.4 \\
$n$ & 4 & 2 & 6 & 7 \\
\hline
\end{tabular}

Values are given as means with $n=$ number of determinations. The clotting mixtures contained $70 \%$ citrated plasma in Tris- $\mathrm{HCl} 10 \mathrm{mM}$, pH 7.4, $\mathrm{NaCl} 150 \mathrm{mM}$, and $\mathrm{CaCl}_{2} 67 \mathrm{mM}$.

expected decrease in cross-linking by plasma factor XIIIa, in that when exhaustively washed in $\left(4^{\circ} \mathrm{C}\right)$ buffer most of the unreduced material migrated as fibrin(ogen) monomer with minor amounts migrating as fibrin dimers and even lesser or trace amounts as oligomers and larger polymers thus explaining the clot solubility in urea. Nevertheless, maximal inhibitory concentrations of C1qI at physiologic temperatures yielded some amount of plasma clot, although minor compared to controls. Thrombin-treated fibrinogen solutions yielded no visible clot unless incubated further at melting ice temperatures when in the presence of $\mathrm{CaCl}_{2}$ a minor granular precipitate formed. When examined in EDTA-containing buffer (not shown) thrombin-induced clot turbidity tended to approach that of non-ClqI controls, but the delayed onset induced by ClqI was invariably unaffected by EDTA $(n=5)$. In binding assays, ${ }^{125}$ I-labeled C1qI bound to immobilized fibrinogen and to $\mathrm{Clq}$, in amounts at least severalfold higher than those bound to albumin or IgG controls $(n=2)$, and this difference was most marked the wash buffer contained Tween 20 (see Methods, $n=5$ ). In a single set of experiments, for example, values of bound ClqI ( $3.5 \mu \mathrm{g}$ applied per microwell) were 29, 113, and $157 \mathrm{cpm} \times 10^{3}$ for albumin, fibrinogen, and $\mathrm{Clq}$, respectively (range $<10 \%$ from each of these means, $n=6$ ). Autoradiograms of $\mathrm{ClqI}$ disclosed the expected size heterogeneity (4), in that six major and at least three minor or trace amount bands ranging from 21 to $\sim 200 \mathrm{kD}$ along with material not entering a $10 \%$ gel were shown. This electrophoretic band pattern was indistinguishable from those displayed by eluates from microwells containing Clq $(n=2)$ or fibrinogen $(n=1)$. In other experiments, immobilized fibrinogen that contained bound ClqI did not bind additional fibrinogen, $(n=4)$, implying that all binding sites on $\mathrm{ClqI}$ had been occupied.

\section{Investigations of the C1qI active moiety}

Enzyme-induced fibrin polymerization. The discovery (15) that the inhibition of the Clq hemolytic activity was attributable to a chromatographic subfraction displaying electrophoretic bands present in the parent material led to experiments that determined an anticoagulant property ${ }^{2}$ (Fig. $1 \mathrm{~A}$ ) indistinguishable from that of the parent material. This chromato-

2. Other subfractions obtained by the gel sieving chromatographic procedure also displayed some inhibition against fibrin gelation, but because their activity against $\mathrm{Clq}$ was minimal or absent these were not investigated further. However, this effect along with their clear binding 'shown by the autoradiograms implied that the anticoagulant effect was not restricted to this chromatographic subfraction. graphic isolate was electrophoretically much less heterogeneous than the parent material (Fig. $2 B$ ), and it was subjected to more extensive investigations. By ${ }^{125}$ I-labeled clot assay, the amounts of coagulable fibrinogen/fibrin in plasma (Fig. $1 B$ ) decreased as concentrations of $\mathrm{Cl} \mathrm{qI}$ were increased. In fibrinogen ( $1 \mu \mathrm{M}, \mathrm{pH} 7.4)$ solutions, the thrombin clotting times were prolonged by the presence of $\mathrm{ClqI}(1 \mu \mathrm{g} / \mathrm{ml})$, from controls of $34.2 \mathrm{~s}$ (range $33.8-35.6 \mathrm{~s}, n=3$ ) to $71.8 \mathrm{~s}(69.3-76.8)$. Also, thrombin-induced clot turbidity decreased progressively with increasing $\mathrm{ClqI}$ concentrations (Fig. $1 \mathrm{D}$ ), and its onset (not shown) was also progressively delayed. Limiting the ClqI concentrations to permit clot formation as shown in Fig. $1 C$ enabled rate measurements of this effect. Using conditions similar to those of Fig. $1 C$ in separate experiments, the rate of rise of clot turbidity was decreased by ClqI from 15 to $4 \times 10^{-3} / \mathrm{s}$. In other experiments, comparisons between ionic strengths of 0.15 and 0.3 disclosed no changes in the extent of inhibition by ClqI $(n=3)$.

In further investigations, the decreased polymerization rate, as well as the turbidity maxima of such clots (Fig. 1 D), were normalized when the ClqI solution had been treated with chondroitinase $\mathrm{ABC}$ ( see Methods, $n=2$ ), consistent with its known proteoglycan structure (6). Moreover, preincubation of ClqI with either a polyclonal anti-ClqI antibody $(n=2$, see Methods, not shown) or with $\mathrm{Clq}$ (Fig. $1 C$ ) did not diminish its anticoagulant effect.

Polymerization of preformed fibrin monomer. Solubilized fibrin (0.5-3 $\mu \mathrm{M})$ induced to polymerize (11) invariably displayed a decreased maximum turbidity in the presence of C1qI and this was also a concentration-dependent effect. In a typical experiment, fibrin added to buffer was $>95 \%$ coagulable but in the presence of $>15$ molar excess of C1qI fibrin failed to form a gel at physiologic, ambient, or melting ice temperatures. Although several or more moles $\mathrm{ClqI} /$ mole fibrinogen were required for marked inhibition of gelation, appreciable inhibition by turbidimetric assay could be shown with as little as 0.3 $\mathrm{mol} / \mathrm{mol}$ fibrin $(n=3)$. The presence of factor XIIIa and $\mathrm{CaCl}_{2}$ had no effect on this incoagulability. Fibrin incoagulable in the presence of $\mathrm{ClqI}$ formed a finely granular precipitate at melting ice temperatures $(n=3)$. Analyzed further, this precipitate amounted to approximately half $(n=2)$ of total protein and readily dissolved on rewarming. As was the case with plasma clots, electrophoretic analyses disclosed that the cryoprecipitate consisted of fibrin monomer, minor amounts of dimers $(\sim 680 \mathrm{kD})$, and of trace amounts of oligomers which migrated into the $3.5 \%$ gel. These oligomers were identical in size (reduced and unreduced) to those of partially crosslinked fibrin controls. Moreover, the cryoprecipitate supernatant contained only monomeric fibrin. Thus by these analyses, no covalent crosslinking of $\mathrm{ClqI}$ to fibrin could be shown.

Fibrin polymerization in the presence of fibrinogen. Numerous attempts to neutralize the ClqI inhibition of fibrin polymerization by preincubation of $\mathrm{ClqI}$ with fibrinogen failed to disclose such an effect. For example, the polymerization rate of $3 \mu \mathrm{M}$ fibrin of $2.3 \times 10^{-2} / \mathrm{s}$ was decreased to 1.5 and 1.3 $\times 10^{-2} / \mathrm{s}$ by fibrinogen $(0.5 \mu \mathrm{M})$ and by ClqI $(9 \mu \mathrm{M})$, respectively. When the two inhibitors were both present in the solution the rate was further decreased to $0.3 \times 10^{-2}$. Similarly, additive inhibition was shown in other experiments $(n=5)$, irrespective of the molar ratio of one inhibitor to an other. Comparison of the calculated slopes of maximal ClqI inhibition before and following its preincubation with fibrinogen 
A

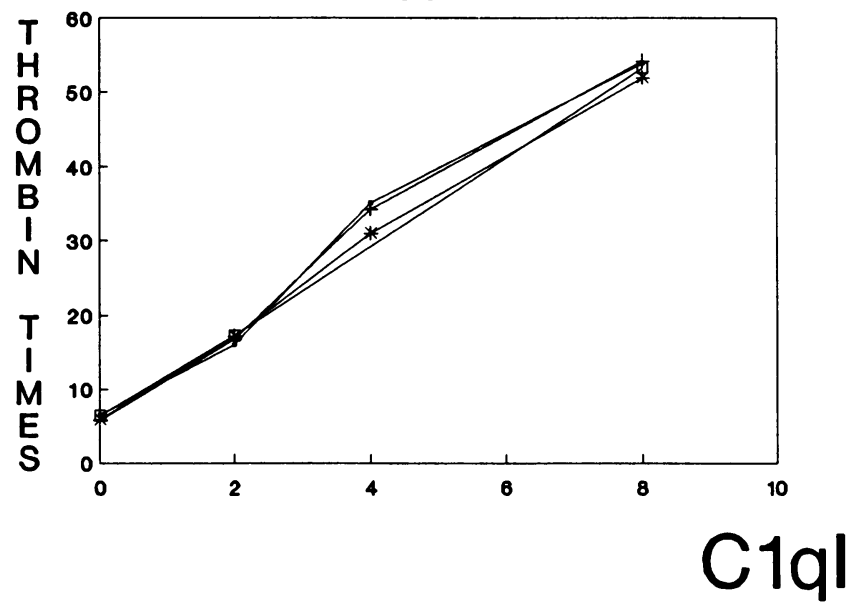

C

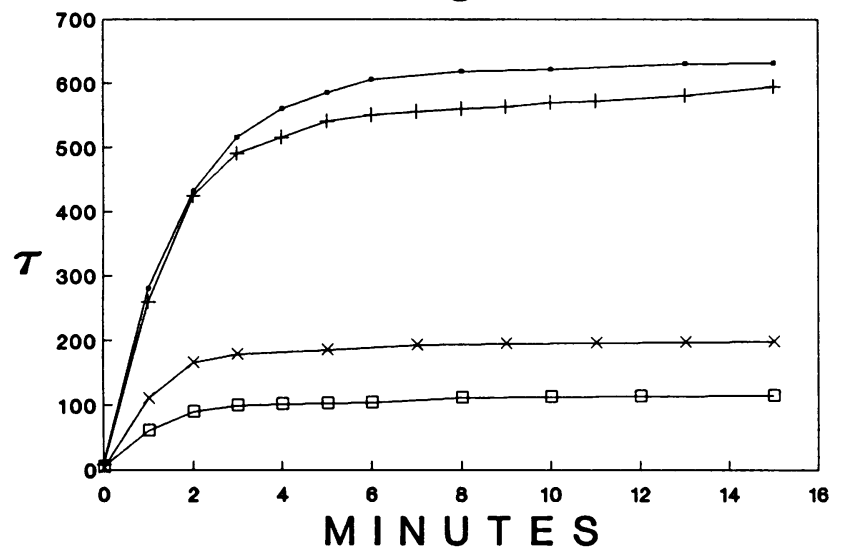

B
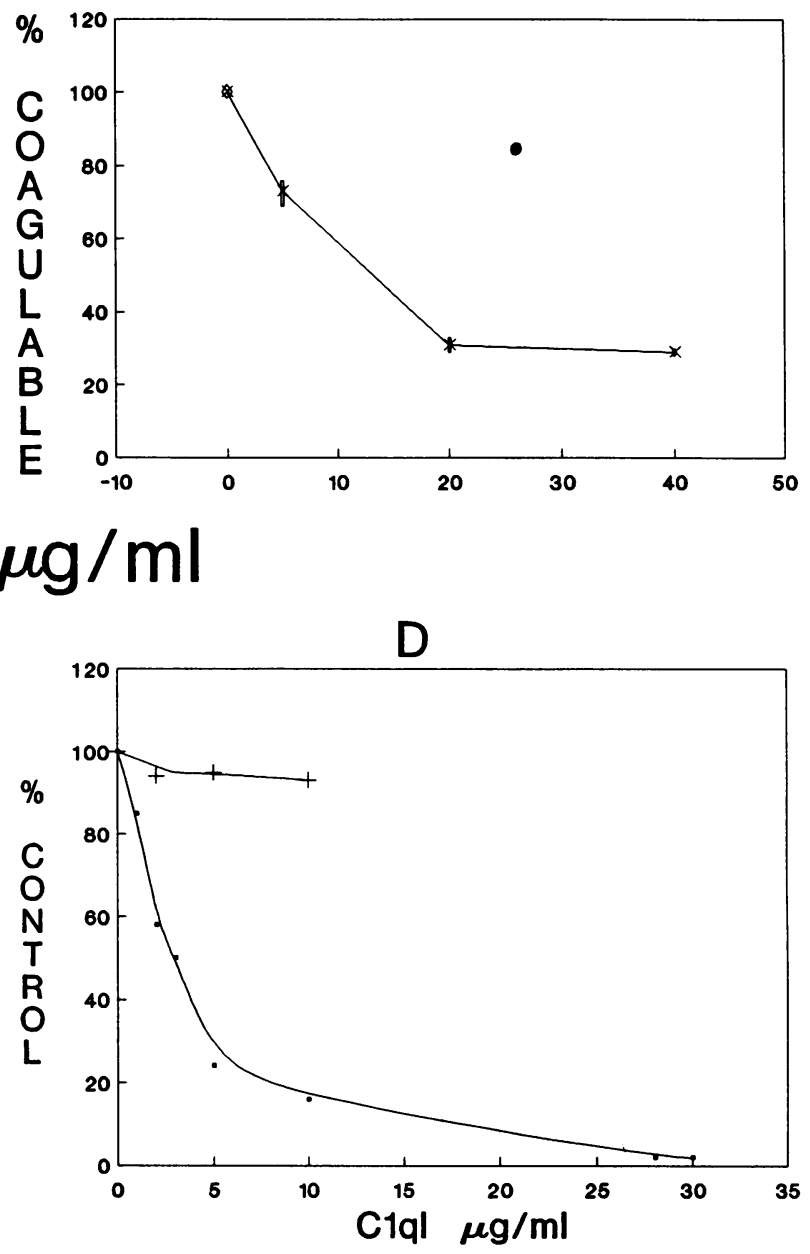

Figure 1. Anticoagulant effects of ClqI. $(A)$ Plasma thrombin times, shown in seconds, of plasma from four donors whose fibrinogen concentrations were $2.2(0), 2.47(+), 2.62(\square)$, and $2.74(*) \mathrm{g} /$ liter, respectively. Each line graph reflects values from the untreated plasma and from plasma to which three different C1qI concentrations shown had been added. $(B)$ Decreased coagulability of fresh citrated plasma, fibrinogen 2.48 $\mathrm{g} /$ liter, to which $\sim 200 \mathrm{ng}{ }^{125}$ I-labeled fibrinogen $/ \mathrm{ml}$ had been added prior to addition of human thrombin. Amounts shown in percentage of total reflect mean and range $(n=3)$ and were calculated from $\mathrm{cpm}$ of the synerized clots and of the clot free liquor. Plasma controls containing $\mathrm{ClqI} 30 \mu \mathrm{g} / \mathrm{ml}$ and lacking added thrombin formed no insoluble gel (not shown). (C) Lack of effect of Clq on the ClqI inhibition of fibrin polymerization, $\mathrm{pH} 7.4, \mu=0.16,37^{\circ} \mathrm{C}$. Thrombin induced turbidity $\left(\tau=\right.$ absorbance, $350 \mathrm{~nm}$, shown $\left.\times 10^{3}\right)$ of fibrinogen $(1.3 \mu \mathrm{M})$ control $(\bullet)$ is compared with that containing ClqI $(\mathrm{x}, 16 \mu \mathrm{g} / \mathrm{ml}), \mathrm{Clq}(+, 84 \mu \mathrm{g} / \mathrm{ml})$, and Clq preincubated ( $90 \mathrm{~min})$ with ClqI (ם). (D) Correction of the effect of $\mathrm{ClqI}(\bullet)$ on the maximum clot turbidity, by ClqI preincubation with chondroitinase $\mathrm{ABC}(+)$. Turbidity maxima (ordinate; see Methods) are expressed as percentage of clots obtained in the absence of ClqI. Fibrinogen, $2.8 \mu \mathrm{M}, 35^{\circ} \mathrm{C}$, was clotted with thrombin, 0.1 $\mathrm{U} / \mathrm{ml}$, and buffer controls, $\mathrm{pH} 7.4, \mu=0.16$, contained inactivated chondroitinase as detailed in methods.

were parallel (Fig. 3), indicating the two inhibitors acted independently against fibrin polymerization. These results were consistent with those from binding experiments (vide infra) and are considered further in the discussion section.

Fibrin(ogen) binding studies. SDS-PAGE autoradiograms of ${ }^{125} \mathrm{I}-\mathrm{ClqI}$ disclosed that, like the parent material, the amounts bound to $\mathrm{Clq}$ as well as those bound to fibrinogen were approximately three- to fivefold higher than those bound to either albumin or IgG controls $(n=5)$. Similarly, the autoradiograms disclosed that eluates from either immobilized fibrinogen or $\mathrm{Clq}$ disclosed the same electrophoretic bands, a major $30-\mathrm{kD}$ and minor bands of 28 and $21 \mathrm{kD}$ (Fig. $2 \mathrm{~B}$ ), thus establishing that the same molecular species possessed the two distinct inhibitory properties, one against $\mathrm{Clq}$ and another against fibrin(ogen). The presence of EDTA ( $2 \mathrm{mM})$ resulted in no change in ClqI binding to either fibrinogen $(n=6)$ or fibrin $(n=6)$. Also, the amounts of $\mathrm{ClqI}$ bound to fibrinogen $(n=6)$ did not change when the ionic strength was increased from 0.15 to 0.5 , consistent with similar results (vide supra) on its inhibition of fibrin polymerization, but in sharp contrast to its known failure to bind to C1q at high ionic strength (4). In further experiments, the polyclonal IgG antibody reacted only

3. These minor ( 21 and $28 \mathrm{kD}$ ) proteoglycan bands appeared as fainter bands in autoradiograms of the parent material (not shown), and were increased in the chromatographic isolate as shown; their band distribution among the three proteins was similar averaging $30 \%, 15 \%$, and $55 \%$ of bound ClqI. Accordingly, for calculating molar amounts bound, a $27-\mathrm{kD} M_{\mathrm{r}}$ mean was employed. Failure of the smaller two bands to react with the polyclonal antibody (i.e., which neutralized 


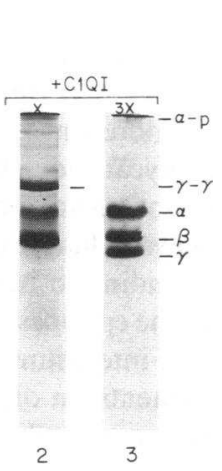

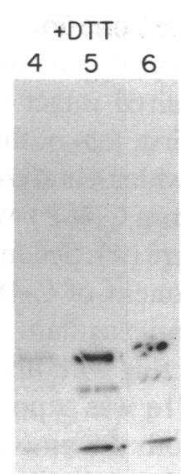

Figure 2. (A) PAGE-SDS comparisons of partly coagulable, gel 2, and incoagulable, gel 3, fibrin obtained at two different concentrations of C: $\mathrm{qI}, 3$ and $9 \mu \mathrm{g} / \mathrm{ml}$ shown as $\times$ and $3 \times$, respectively, and obtained under maximum cross-linking conditions. Gel 1 , fibrin clot contr l) $\beta$ chains of fibrin, $\alpha-p, \alpha$ polymers; $\gamma-\gamma, \gamma-\gamma$ dimers. Clotting conditions were $2 \mu \mathrm{M}$ fibrinogen, $40 \mathrm{nM}$ XIIIa, and $5 \mathrm{mM} \mathrm{CaCl}_{2}$, $0.1 \mathrm{U}$ thrombin $/ \mathrm{ml}, 50 \mathrm{U} \mathrm{KPTI} / \mathrm{ml}, \mathrm{pH} 7.4, \mu=0.15$, incubated at ambient temperature overnight. $(B)$ Comparison of autoradiograms of ${ }^{125} \mathrm{I}-\mathrm{C} 1 \mathrm{qI}$ eluates from microwell immobilized human albumin, lanes 1 and 4, Clq, lanes 2 and 5 , and fibrinogen, lanes 3 and 6 ; lanes $1-3$ reflect unreduced and lanes 4-6 reduced material. The band between that of 30 and that of $21 \mathrm{kD}$ was $28 \mathrm{kD}$. Identical amounts of $\mathrm{Cl}$ qI were applied ( $3.5 \mu \mathrm{g} /$ microwell) and each lane reflects amounts bound to four microwells and eluted by the urea-SDS sample buffer. Calculated from a densitometric scan, of the total bound ClqI, $47 \%$ bound to $\mathrm{Clq}, 42 \%$ to fibrinogen, and $11 \%$ to albumin; for $\mathrm{mol} / \mathrm{mol}$ fibrinogen and Clq estimates see Table II and legend.

against the 30-kD band, in Western blot experiments (not shown ). ${ }^{3}$ Preincubation of $\mathrm{ClqI}$ with either chondroitin- 4 sulfate (i.e., a polysaccharide component of C-4SP, $n=2$ ) or Clq in molar excess $(n=4)$ did not diminish the amount of ClqI bound to fibrinogen whether or not it had been pretreated with $\mathrm{Clq}$ or with chondroitin sulfate, respectively. This intimated that native $\mathrm{ClqI}$ structure was required for its binding to fibrinogen, and that the Clq and fibrinogen binding sites on ClqI were distinct. In further analyses, ClqI exposure to chondroitinase $\mathrm{ABC}$ decreased its binding to fibrinogen to that of albumin controls $(n=6$; not shown $)$.

Comparison of $\mathrm{ClqI}$ binding by immobilized fibrinogen, monomeric desAA fibrin, and desAA/desBB fibrin (Table II), disclosed ClqI binding to all three forms but in respectively decreased amounts. Also, fibrin $(1 \mu \mathrm{M})$ clots immersed overnight in $6.7 \mu \mathrm{M} \mathrm{ClqI}$ bound $68 \%$ of ClqI $(n=4)$, indicating that $\mathrm{ClqI}$ binding sites remained available in polymerized fibrin. These results led to assessment of binding to isolated plasmic fragment $\mathrm{E}$ of fibrinogen. Fragment $\mathrm{E}$ also bound $\mathrm{ClqI}$ (Table II), but this was virtually abolished by prior exposure of fragment $E$ to thrombin, indicating peptide $A$ was necessary for binding. Fragment $E$ contains decreased amounts of intact $B \beta$ chains (18) precluding B peptide assessments; however, a role

$\mathrm{Clq}$ inhibitory activity, vide supra) suggested they were species with no inhibitory activity against Clq. Alternatively, they were inhibitory but lacked the antigen epitope possibly reflecting either fragments of a larger (e.g., $30 \mathrm{kD}$ ) species or other unrelated forms. Nevertheless, they typically did not stain with Coomassie Blue and were assumed to be $\mathrm{C}-4 \mathrm{SP}$ in accord with the known characterization of the parent material (4).

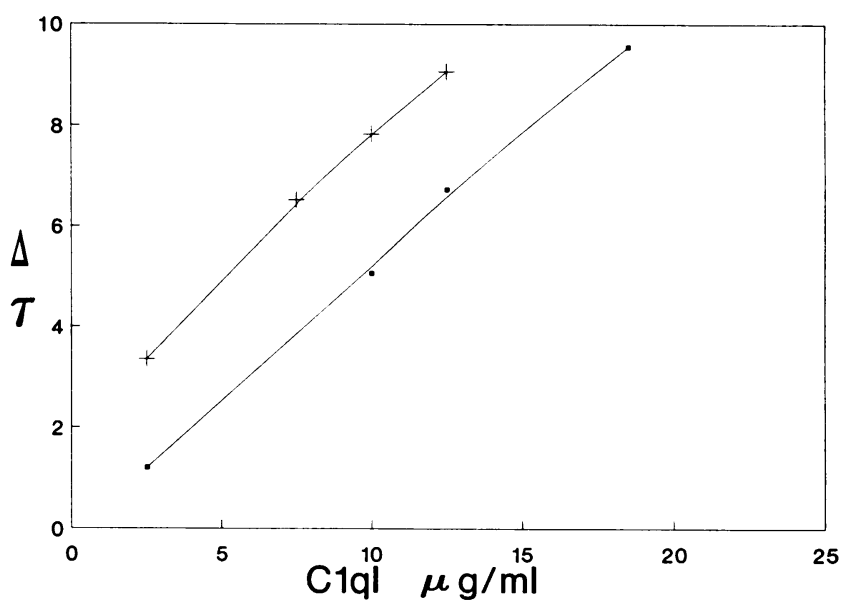

Figure 3. Inhibition of polymerization of $(2 \mu \mathrm{M})$ fibrin monomer by $\mathrm{ClqI}$ in the presence $\mathrm{x}$ ) and absence $(\odot)$ of $(1.5 \mu \mathrm{M})$ fibrinogen at different ClqI concentrations (abscissa). $\Delta \tau$, change in maximum turbidity (extent of decreased absorbance, $350 \mathrm{~nm}$, shown $\times 10^{3}$ ). The experiments were carried out at $37^{\circ} \mathrm{C}, \mathrm{pH} \mathrm{7.4, \mu}=0.15$.

of the uncleaved $B$ peptide was clearly indicated by comparisons of ClqI binding to dessAA and desAA/desBB fibrin monomers ( Table III), and by results indicating cleaved B peptide bound to liquid phase ClqI (vide infra). To explore the $\mathrm{ClqI}$ binding to the intact $\mathrm{E}$ domain, immobilized fibrinogen containing bound ${ }^{125} \mathrm{I}-\mathrm{Clq} \mathrm{I}$ was exposed to thrombin $(1 \mathrm{U} / \mathrm{ml}$, overnight $)$. This resulted in no release of ClqI $(n=6)$. Also, ${ }^{125}$ I-thrombin was exposed to fibrinogen containing bound ClqI $(n=6)$, and the amounts of thrombin bound, did not differ from those bound to fibrinogen controls whether or not thrombin had been inactivated by P-PACK.

Binding to fragment $D_{1}$ was also demonstrated (Table II). This and the lack of binding to thrombin treated $E$ suggested that binding to fibrin monomer reflected binding to the intact $D$ domain. In related investigations, the amounts of either radiolabeled fragment $D_{1}$ or fibrinogen bound by solid-phase fibrin monomers were undiminished following binding of $\mathrm{ClqI}$ to such monomers $(n=6)$. This was consistent with the lack of

Table II. Amounts of ClqI Bound to Fibrin Monomers and Polymers, and Isolated Plasmic Fibrinogen Fragments $D_{1}$ and $E$

\begin{tabular}{|c|c|c|c|c|c|c|}
\hline \multirow[b]{2}{*}{ Fibrinogen } & \multirow{2}{*}{$\begin{array}{c}\text { Fibrin } \\
\text { clot }\end{array}$} & \multicolumn{2}{|c|}{ Fibrin monomer } & \multicolumn{2}{|c|}{ Fragment } & \multirow[b]{2}{*}{ E-Thr. } \\
\hline & & $\operatorname{des} A A$ & $\operatorname{des} A \mathrm{~A} / \operatorname{des} B \mathrm{~B}$ & $\mathrm{D}_{1}$ & E & \\
\hline \multicolumn{7}{|c|}{$\mathrm{mol} / \mathrm{mol}$} \\
\hline 6.7 & 5.3 & 5.6 & 3.7 & 1.9 & 3.5 & $<1$ \\
\hline
\end{tabular}

Shown are means (range $<8 \%$ above and below each mean) from at least triplicate measurements using varying ratios of radiolabeled/unlabeled ClqI, corrected for albumin controls. Not shown were Clq-positive controls disclosing $16 \mathrm{~mol} \mathrm{ClqI}$ bound/mol Clq Amounts in fibrin polymers were computed from synerized fibrin (1 $\mu \mathrm{M})$ clots and their supernatants described in Methods, using a ClqI concentration of $7 \mu \mathrm{M}$. All others reflect amounts bound by microwell immobilized ligand as described in Methods. Abbreviations: fibrin monomer desAA, fibrin lacking both A peptides; fibrin monomer desAA/desBB, fibrin lacking both $A$ and both B peptides; E-Thr, fibrinogen fragment $\mathrm{E}$ treated with thrombin before $\mathrm{ClqI}$ exposure. 
Table III. Fibrinopeptide Binding to CIqI

\begin{tabular}{|c|c|c|c|c|}
\hline $\mathrm{ClqI}(\mu g / m l)$ & 3 & 10 & 20 & 40 \\
\hline & \multicolumn{4}{|c|}{$\%$} \\
\hline A Peptide & 87 & 57 & 40 & 0 \\
\hline B Peptide & 94 & 80 & 53 & 18 \\
\hline
\end{tabular}

Values reflect unbound peptides calculated as percentage of A and B peptide controls from HPLC measurements. The ClqI + peptide mixture was assayed after microfiltration described in Methods. Estimated from these and related measurements, not shown, at least 1 mol peptide A, and 0.7 mol peptide B were bound by $1 \mathrm{~mol}$ of ClqI.

binding of $\mathrm{ClqI}$ to the fibrin $\mathrm{E}$ domain and with other results (vide supra) disclosing additive inhibition of fibrin polymerization by fibrinogen and ClqI.

Binding to fibrinopeptides. Preliminary attempts to assess possible effects of ClqI on A and B peptide release failed ( $n$ $=4$ ), and this was further explored by preparing mixtures of cleaved A and B peptides with ClqI in molar excess. These yielded no detectable free peptides by HPLC measurements. For example, a solution containing $\sim 10 \mu \mathrm{g} \mathrm{ClqI} / \mathrm{ml}, 2 \mu \mathrm{g}$ of $\mathrm{A}$, and $2 \mu \mathrm{g}$ of $\mathrm{B} / \mathrm{ml}$ yielded no free $\mathrm{A}$ or B peptides $(n=3)$. That is, when such solution mixtures were applied whole or after microfiltration to remove ClqI (see Methods), no free peptides were detectable in the filtrate, in sharp contrast to control solutions lacking ClqI. Moreover, the amounts of free peptides were inversely proportional to the $\mathrm{ClqI}$ concentration (Table III). Also, using a single A and B peptide solution mixture higher $\mathrm{ClqI}$ concentrations were required to remove peptide $\mathrm{B}$ than those which removed peptide $\mathrm{A}$ or $\mathrm{AP}(n=3)$, peptide AP requiring the lowest concentrations of ClqI for removal (not shown). Calculated from such data, a single mole of ClqI displayed a capacity to bind at least $1 \mathrm{~mol}$ of peptide A and $0.7 \mathrm{~mol}$ of $B$ from the same mixture. Since the amounts of AP peptide were minor (i.e., $<20 \%$ of A) this was not separately calculated.

\section{Discussion}

The most important findings of the foregoing investigations relate to the capacity of C1qI to bind to fibrin (ogen) either free and in complex with Clq and to impair fibrin gelation in plasma. The plasma results indicated that $\mathrm{ClqI}$ binding to fibrinogen was selective among other proteins, shown by the binding of much lower amounts to isolated albumin and IgG. Moreover, the circulating form of (i.e., Clqrs complex) does not bind ClqI (7). Because most circulating proteoglycans are protein bound $(2,4)$ our results suggest that fibrinogen is a major binding protein in normal plasma; anticoagulant effects by such proteoglycans could not be expected owing to their low

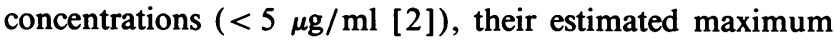
binding of $6.7 \mathrm{~mol} / \mathrm{mol}$ fibrinogen (vide supra), the at least 20-fold molar excess of fibrinogen, and some amounts bound by other proteins. That the same molecule(s) expressed the two inhibitory effects was established by the identical electrophoretic bands of eluates from fibrinogen and from Clq. Also, fibrinogen bound ClqI could bind no additional fibrinogen. Absent a self-association by ClqI that may mask such epitopes, this implied a single set of epitopes for fibrin(ogen) binding (i.e., one for $\mathrm{E}$ and one for $\mathrm{D}$ domain sites, respectively). A related conclusion, that the fibrinogen interacting epitope required intact ClqI was implied by two sets of experiments. First, loss of this property by exposure to chondroitinase $A B C$ (which catalyzes cleavage of galactosaminoglycan side chains from C-4SP) was consistent with its known proteoglycan structure (4). Second, chondroitin-4 sulfate (a polysaccharide component of C-4SP) diminished neither the binding to nor the anticoagulant effect against fibrinogen. That the epitopes interacting with fibrinogen were distinct from that interacting with Clq was supported by three series of experiments. In one set, both the amounts of ClqI bound to fibrinogen as well as the related anticoagulant effect remained undiminished by its preincubation with $\mathrm{Clq}$ under conditions which neutralized the Clq hemolytic activity. In an other set, our polyclonal IgG antibody abolished its capacity to neutralize the $\mathrm{Clq}$ hemolytic activity, but it had no effect on the anticoagulant property of ClqI. Finally, neither the anticoagulant property of ClqI nor its binding to fibrinogen were diminished by increased ionic strength in sharp contrast with its binding to $\mathrm{Clq}$ which is abolished by high ionic strength (4).

The results imply two independent but concerted mechanisms of action by ClqI. One mechanism involves binding of $\mathrm{ClqI}$ to the fibrinogen $\mathrm{E}$ domain; at least $2 \mathrm{~mol}$ of C1qI bound to the $\mathrm{E}$ domain before but not after fibrinopeptides have been cleaved, assuming no self-associating C1qI complexes. Fibrinopeptide cleavage was more difficult to assess. Failure to release bound ClqI by thrombin suggested impaired fibrinopeptide cleavage. However, even under maximum ClqI inhibition some fibrin monomer formed in both plasma and fibrinogen solutions (i.e., insoluble material in plasma, and granular aggregates in isolated fibrinogen solutions showing $\gamma-\gamma$ dimers, could be harvested by prolonged incubation at melting ice temperatures). This implied that some cleavage of fibrinopeptides occurred, however slow or partial, although these were not assayed owing to their binding to ClqI. It is unclear whether multiple epitopes or a single ClqI epitope bound fibrinopeptides. Differences in binding of each fibrinopeptide by ClqI suggested that similar C1qI sites bound fibrinopeptides but with different affinities owing to peptide structure differences. The dessAA data (Table III) intimated that one mol of ClqI bound to desAA, two to fibrinogen and none to the desAA/ dessBB fibrin $E$ domain. This along with the capacity for binding nearly one $B$ and one A peptide implied two distinct binding epitopes for the $\mathrm{E}$ domain. The apparent higher affinity for the AP peptide suggests subterminal carbohydrate structures are involved rather the terminal sialic acid residues. Also of interest, neither native nor P-PACK neutralized thrombin binding to fibrinogen was diminished by ClqI occupancy. This raises the possibility that fibrinogen-immobilized ClqI itself bound thrombin, consistent with the demonstrated failure of thrombin to release any bound C1qI. ClqI clearly requires uncleaved $A$ and to a lesser extent $B$ for its occupancy of the $E$ domain, and this makes it difficult to envision unhindered binding of thrombin to at least the catalytic site on $\mathrm{E}$.

A second mechanism, emerged from the fibrin polymerization and binding studies, both supporting the conclusion that $\mathrm{D}$ domain polymerization sites were not occupied by $\mathrm{ClqI}$ and that $\mathrm{Clq}$ I bound elsewhere within the $\mathrm{D}$ domain and inhibited by steric hindrance. This was supported by $(a) \mathrm{ClqI}$ binding to fibrin monomers and to $\mathrm{D}_{1},(b)$ the incorporation of ClqI in polymerized fibrin gels, $(c)$ the capacity of fibrinogen or of 
fragment $D_{1}$ to inhibit fibrin polymerization in the presence of ClqI, and $(d)$ the additive inhibition of polymerization by fibrinogen and ClqI (Fig. 3). Additional evidence of steric hindrance was provided by use of $\mathrm{ClqI}$ concentrations which allowed partial insoluble gel formation. All $\gamma$ chains formed $\gamma-\gamma$ dimers (Fig. $2 A$ ) indicating complete oligomer or protofibril formation. However, little or no $\alpha$-polymers formed, shown in Fig. $2 A$, indicating progression to more complex polymer forms could not be demonstrated. This implied that occupancy of fibrin $\mathrm{E}$ by the $\mathrm{D}$ domains of two other monomers (i.e., linear polymerization ) was not impaired, notwithstanding that it may have occurred slowly. However, lateral or $\alpha$ chain dependent polymerization was either prevented or possibly occurred by misalignment which prevented formation of covalent crosslinks containing $\alpha$ chains.

Unimpaired thrombin binding along with the potent effect against fibrin polymerization by $\mathrm{ClqI}$, raises possible in vivo implications. It may, for example, provide an enhancement of the anticoagulant state of the relatively self-contained inflammatory or tissue repair microenvironments in the presence of saturated inhibitors such as AT III. Even where limited concentrations of fluid phase $\mathrm{ClqI}$ exist whether or not it is bound to $\mathrm{Clq}$ /antibody complexes, $\mathrm{ClqI}$ does bind to fibrinogen and to fibrin serving to further limit thrombogenesis. Clearly, such complexed fibrin(ogen) still binds thrombin serving to further enhance the anticoagulant state. Furthermore, such binding to immune complexes may explain the long known presence of fibrin(ogen) in immune complex-related pathologic lesions. Reported binding of Clq to fibrinogen (19) appears to be independent of the $\mathrm{ClqI}$ effect. That is to say, neither the anticoagulant effects nor the binding of $\mathrm{ClqI}$ could be diminished by its preincubation of or in the presence of molar excess of $\mathrm{Clq}$ (vide supra). In that report a different $\mathrm{Clq}$ isolation procedure was used and it is unclear whether a possible effect by undesorbed ClqI played a role, if any. Whether or not membrane intercalated or other solid-phase proteoglycans bind fibrinogen is an open question. A report on thrombomodulin (20), a thrombin inhibitor residing on endothelial cell membranes, is of interest in this regard, in that enzymic removal of the sulfated GAG moiety of rabbit thrombomodulin abolished its anticoagulant activity against thrombin fibrinogen clotting, intimating an effect similar to that of the present proteoglycan.

Among other possible in vivo roles suggested by the results, one relates to cell receptor binding of fibrinogen. Certain fluidphase chondroitin sulfate proteoglycans reportedly (5) inhibit receptor interaction with ligand, particularly as it relates to extracellular matrix proteins, or they may inhibit cell-cell interaction (21). Conversely, membrane intercalated proteoglycans mediate cell-matrix and cell-cell interactions; melanoma cells, for example, failed to interact with the extracellular matrix when their chondroitin sulfate proteoglycan synthesis was blocked (22), leading the authors to suggest a cooperative role between cell receptors and such membrane proteoglycans. Since fibrinogen and fibrin are native occupants of such extracellular sites early on during thrombus repair, their interaction with cell membrane proteoglycans may play a role in receptor mediated cell transit within the thrombus.

A mechanism for the presence of fibrinogen in atherosclerotic lesions (23-26) can be formulated from our results. During early stages of the endothelial lesion or loss, exposed basement membrane being rich in proteoglycans (5) can bind fibrinogen from circulation thus preventing its fibrinopeptide release even though it does not prevent thrombin binding (present studies). Thus, fibrinogen remains in atheromata (23-26) and in the intima adjoining such or other conditions $(23,26)$. Similarly, a possible role by such proteoglycans in thrombus formation and function can also be postulated. By tight binding to the basement membrane proteoglycans, the fibrin polymer is densely anchored, and this enhances its (noncovalent) stability and hemostatic effectiveness. Finally, the present findings raise the possibility of fibrinopeptide binding by proteoglycans in blood or tissues thus making them inaccessible to the widely used fluid-phase measurements. Assuming such fluid phase binding does not impair their immunoassay (27), binding by solid-phase proteoglycans, may influence their circulating levels and possibly account for the very short half life of fibrinopeptides.

The possible relationship of $\mathrm{ClqI}$ to circulating proteoglycans, which are increased in some disorders (2) and display anticoagulant properties in others (28-32), is speculative, in that attempts to assay for a C1qI inhibitory activity were not made. Nevertheless, among this heterogeneous group chondroitin proteoglycans have been described $(28,34)$. Most glycosaminoglycans identified as circulating anticoagulants enhanced the antithrombin III activity and thus were directed against thrombin; when examined further, evidence for heparan (29), and keratan $(29,34)$ sulfate proteoglycans and for hyaluronic acid (30) was obtained in different patients. A chondroitin sulfate proteoglycan (28) differed from the others by impairing the thrombin release of fibrinopeptides, suggesting some similarity to the present form. These and other reported cases implied association with substantial neoplastic proliferation (28-34) and with parenchymal tissue destruction $(31,32)$. Even so, keratan sulfate along with dermatan sulfate are known to be increased in mucopolysaccharide storage diseases but are not associated with a coagulopathy (33). Increased circulating levels of proteoglycans occur in suramin treated patients (34) and among these, undersulfated chondroitin sulfate forms not unlike the present proteoglycan have been described. Along with abnormal accumulation of proteoglycans in the liver of suramin-treated rats (35), these findings intimated inhibition of enzymes which degrade proteoglycans. Thus, catabolic processes, as well as cell proliferation and tissue destruction appear to modulate their circulating levels in health and disease.

\section{Acknowledgments}

The authors wish to thank Dr. B. Coller for his generous support and Yuqun Hong for excellent technical assistance.

The foregoing studies were supported in part by grants CA-4107 from the National Cancer Institute and 900696 from the American Heart Association.

\section{References}

1. Doolittle, R. F. 1984. Fibrinogen and fibrin. Annu. Rev. Biochem. 53:195229.

2. Friman, C., D. Nordstrom, and I. Eronen. 1987. Plasma glycosaminoglycans in systemic lupus erythematosus. J. Rheumatol. 14:1132-1134.

3. Jackson, R. L., S. J. Busch, and A. D. Cardin. 1991. Glycosaminoglycans: molecular properties, protein interactions, and role in physiologic processes. Phys iol. Rev. 71:481-539.

4. Silvestri, L., J. R. Baker, L. Roden, and R. M. Stroud. 1981. The Clq inhibitor in serum is a chrondroitin 4-sulfate proteoglycan. J. Biol. Chem. 256:7383-7387. 
5. Ruoslahti, E. 1989. Proteoglycans in cell regulation. J. Biol. Chem. 265:13369-13372.

6. Conradie, J. D., J. E. Volanakis, and R. M. Stroud. 1975. Evidence for a serum inhibitor of Clq. Immunochemistry. 12:967-971.

7. Ghebrehiwet, B., L. Silvestri, and C. McDevitt. 1984. Identification of the Raji cell membrane-derived Clq inhibitor as a receptor for human Clq: purification and immunochemical characterization. J. Exp. Med. 160:1375-1389.

8. LeBoeuf, R. D., R. G. Gregg, P. H. Weigel, and G. M. Fuller. 1987. Effects of hyaluronic acid and other glycosaminoglycans on fibrin polymer formation. Biochemistry. 26:6052-6057.

9. Carr, M. E., and P. L. Powers. 1989. Effect of glycosaminoglycans on thrombin- and atroxin-induced fibrin assembly structure. Thromb. Haemostasis. 62:1057-1061.

10. Mosesson, M. W., and S. Sherry. 1966. The preparation and properties of human fibrinogen of relatively high solubility. Biochemistry. 5:2829-2835.

11. Galanakis, D. K., B. P. Lane, and S. R. Simon. 1987. Albumin modulates lateral assembly of fibrin polymers: evidence of enhanced fine fibril formation and of unique synergism with fibrinogen. Biochemistry. 26:2389-2400.

12. Weber, K., and M. J. Osborn. 1975. Proteins and sodium sulfate: molecular weight determination on polymacrylamide gels and related procedures. In The Proteins, 3rd edition. H. Neurath and R. H. Hill, editors. Academic Press, Inc. New York. 179-223.

13. Lorand, L., T. Urayama, J. W. C. de Kiewiet, and H. L. Nossel. 1969. Diagnostic and genetic studies on fibrin-stabilizing factor with a new assay based on amine incorporation. J. Clin. Invest. 48:1054-1064.

14. Kehl, M., F. Lottspeich, and A. Henschen. 1981. Analysis of human fibrinopeptides by high performance liquid chromatography. Hoppe-Seylers's Z. Physiol. Chem. 362:1661-1664.

15. Bossone, S. A., and B. Ghebrehiwet. 1987. Further characterization of compliment Clq inhibitor. Complement. 123:137. (Abstr.)

16. Kolb, W. P., L. M. Kolb, and E. R. Podack. 1979. Clq: isolation from human serum in high yield by affinity chromatography and development of a highly sensitive hemolytic assay. J. Immunol. 122:2103-2111.

17. Ghebrehiwet, B. 1981. Clq inhibitor (ClqINH): Functional properties and possible relationship to a lymphocyte membrane associated $\mathrm{Clq}$ precipitin. J. Immunol. 126:1837-1842.

18. Kowalska-Loth, B., B. Gardlund, N. Edgberg, and B. Blomback. 1973. Plasmic degradation products of human Fibrinogen. II. Chemical and immunologic relation between E and N-DSK. Thromb. Res. 2:423-450.

19. Entwistle, R. A., and L. T. Furcht. 1988. Clq component of complement binds to fibrinogen and fibrin. Biochemistry. 27:507-512.

20. Bourin, M.-C., and U. Lindhal. 1990. Functional role of the polysaccharide component of rabbit thrombomodulin: effects on the inactivation of thrombin by antithrombin, cleavage of fibrinogen by thrombin, and thrombin-catalyzed activation of factor V. Biochem. J. 270:419-425.

21. Minguell, J. J., C. Hardy, and M. Tavassoli. 1992. Membrane-associated chrondroitin sulfate proteoglycan and fibrinonectin mediate the binding of hemopoietic progenitor cells to stromal cells. Exp. Cell Res. 201:200-207.

22. lida, J., A. P. N. Skubitz, L. T. Furcht, E. A. Wayner, and J. B. McCarthy. 1992. Co-ordinate role for cell surface chondroitin sulfate proteoglycan and $\alpha 4 \beta 1$ integrin in mediating melanoma cell adhesion to fibronectin. J. Cell Biol. 118:431-444.

23. Shainoff, J. R., and I. H. Page. 1972. Deposition of modified fibrinogen with the aortic intima. Atherosclerosis. 16:287-305.

24. Smith, E. B., K. M. Alexander, and I. B. Massie. 1976. Insoluble "fibrin" in human aortic intima. Quantitative studies on the relationship between insoluble "fibrin," soluble fibrinogen and low density lipoprotein. Atherosclerosis. 23:19-39.

25. Bini, A., J. Fenoglio, Jr., J. Sobel, J. Owen, M. Fejgl, and K. L. Kaplan. 1987. Immunochemical characterization of fibrinogen, fibrin I and fibrin II in human thrombi and athersosclerotic lesions. Blood. 69:1038-1045.

26. Valenzuela, R., J. R. Shainoff, P. M. Di Bello, D. A. Urbanic, J. M. Anderson, G. R. Matsueda, and B. J. Kudryk. 1992. Immunoelectrophoretic and immunohistochemical characterizations of fibrinogen derivatives in atherosclerotic aortic intimas and vascular prostheses pseudo-intimas. Am. J. Pathol. 141:861-880.

27. Nossel, H. L., I. Yudelman, R. E. Canfield, V. P. Buttler, Jr., K. Spanondis, G. D. Wilner, and G. D. Qureshi. 1974. Measurement of fibrinopeptide A in human blood. J. Clin. Invest. 54:43-53.

28. Liebman, H. A., R. Comenzo, S. T. Allen, and J. R. Dilorio. 1991. A glycosaminoglycan inhibitor of thrombin: a new mechanism for abnormal hemostatic assays in cancer. Am. J. Hematol. 38:24-29.

29. Kaufman, P. A., J. P. Gockerman, and C. S. Greenberg. 1989. Production of a novel anticoagulant by neoplastic cells: report of a case and review of the literature. Am. J. Med. 86:612-616.

30. Rodgers, G. M., and L. Corash. 1985. Acquired heparin-like anticoagulant in a patient with metastatic breast carcinoma. West. J. Med. 143:672-675.

31. Rick, M., and H. Gralnick. 1984. Circulating heparin-like anticoagulants. N. Engl. J. Med. 311:1056.

32. Boinot, C., J. Martin, and M. Larregue. 1986. Heparin-like activity, atopic eczema, and contact dermatitis. Ann. Intern. Med. 105:302.

33. McKusick, V. A., and E. Neufeld. 1983. The mucopolysaccharide storage diseases. In The Metabolic Basis of Inherited Disease. 5th edition. J. B. Standbury, J. B. Wyngaarden, D. S. Fredrickson, J. L. Goldstein, and M. S. Brown, editors. McGraw-Hill, Inc., New York. 751.

34. McDonald, K. H., C. A. Stein, V. R. LoRocca, and C. E. Myers. 1988. Circulating glycosaminoglycan anticoagulants associated with suramin treatment. Blood. 71:273-279.

35. Constantopoulos, G., S. Rees, B. G. Cragg, J. A. Barranger, and R. O. Brady. 1980. Experimental animal model for mucopolysaccharidosis: Suramininduced glycosaminoglycan and sphingolipid accumulation in the rat. Proc. Natl. Acad. Sci. USA. 77:3700-3704. 